\title{
Further insight into crystal structures of E. coli IspH/LytB in complex with two potent inhibitors of the MEP pathway: a starting point for rational design of new antimicrobials
}

\author{
Franck Borel ${ }^{[\mathrm{a}] *}$, Elodie Barbier ${ }^{[\mathrm{a}]}$, Sergiy Krasutsky ${ }^{[\mathrm{b}]}$, Karnjapan Janthawornpong ${ }^{[\mathrm{c}]}$, Philippe Chaignon ${ }^{[\mathrm{c}]}$, \\ C. Dale Poulter ${ }^{[b]}$, Jean-Luc Ferrer ${ }^{[a]}$ and Myriam Seemann ${ }^{[c] *}$
}

\begin{abstract}
$\mathrm{IspH}$, also called LytB, a protein involved in the biosynthesis of isoprenoids via the methylerythritol phosphate pathway, is an attractive target for the development of new antimicrobial drugs. Here, we report crystal structures of Escherichia coli IspH in complex with the two most potent inhibitors known to date, $(E)$-4-mercapto-3methylbut-2-en-1-yl diphosphate (TMBPP) and (E)-4-amino-3methylbut-2-en-1-yl diphosphate (AMBPP) at $1.95 \AA$ and $1.7 \AA$ resolution, respectively. The structure of the $E$. coli IspH:TMBPP complex exhibited two conformers of the inhibitor. This unexpected feature has been exploited to design and evolve in silico new antimicrobial candidates.
\end{abstract}

\section{Introduction}

Isoprenoids are found in all living organisms and belong to a structurally diverse family of natural products. They are formed by the addition of one or more molecules of isopentenyl diphosphate (IPP, 1) to dimethylallyl diphosphate (DMAPP, 2). Surprisingly, these fundamental building blocks are synthesized by two quite different routes: the mevalonate pathway ${ }^{[1]}$ and the methylerythritol phosphate (MEP) pathway. ${ }^{[2,3]}$ The mevalonate pathway is distributed among archaebacteria, a few eubacteria and most eukaryotes. The MEP pathway occurs in most bacteria including Mycobacterium tuberculosis, in parasites, for example Plasmodium falciparum responsible for malaria, and in plant chloroplasts. This pathway is absent in humans but essential for microorganisms. Given the severe threat from organisms resistant to conventional antibacterial agents, targeting the MEP pathway is an attractive strategy in the search for new antimicrobial agents. ${ }^{[4-6]}$

The MEP pathway transforms pyruvate 3 and D-glyceraldehyde phosphate 4, via several intermediates (5-10), into IPP 1 and DMAPP 2 (Scheme 1). The last step of this biosynthetic route,

[a] Dr. F. Borel, E. Barbier, Dr. J-L. Ferrer Institut de Biologie Structurale IBS, Univ. Grenoble Alpes, CEA CNRS, 38044 Grenoble, France.

E-mail: franck.borel@ibs.fr

[b] Dr. S. Krasutsky, Dr. C. D. Poulter

Department of Chemistry, University of Utah, 315 South 1400 East RM 2020, Salt Lake City, Utah 84112, United States

[c] Dr. K. Janthawornpong, Dr. P. Chaignon, Dr. M. Seemann Université de Strasbourg, CNRS, CHIMIE UMR 7177, F-67000 Strasbourg, France.

E-mail: mseemann@unistra.fr conversion of (E)-4-hydroxy-3-methylbut-2-en-1-yl diphosphate (HMBPP, 10) into a mixture of IPP 1 and DMAPP 2, is catalyzed by IspH (EC: 1.17.1.2), also called LytB. This oxygen-sensitive protein harbors a peculiar $[4 \mathrm{Fe}-4 \mathrm{~S}]^{2+}$ center, with one iron linked to three inorganic sulfur atoms from the cluster and to two or three non-sulfur ligands ( $\mathrm{N}$ and/or $\mathrm{O}$ ), as shown by Mössbauer spectroscopic studies of the substrate-free enzyme. ${ }^{[7]}$ Very recently, synchrotron-based nuclear resonance vibrational spectroscopy (NRVS) experiments, a technique that specifically senses vibrations involving iron, in combination with quantum chemical - molecular mechanical (QM/MM) calculations provided evidence that these unknown ligands are three water molecules. ${ }^{\left[{ }^{[8}\right.}$ The first X-ray structures reported for IspH from Aquifex aeolicus ${ }^{\left[{ }^{[9]}\right.}$ and Escherichia coli ${ }^{[10]}$ contained an incomplete [3Fe-4S] cluster. No crystal structure of substrate-free IspH in its $[4 \mathrm{Fe}-4 \mathrm{~S}]^{2+}$ form has been reported. The inability to obtain such a structure is most probably due to the instability of the apical iron linked to three water molecules, which dissociates during the crystallization process. A structure of $E$. coli IspH with an intact [4Fe-4S] cluster was obtained as a complex with the substrate HMBPP $10 .{ }^{[11]}$ Here, we describe the structures of $E$. coli IspH in complex with the two most potent inhibitors of the enzyme (Scheme 2): (E)-4-mercapto3-methylbut-2-en-1-yl diphosphate (TMBPP, 11) and $(E)$-4amino-3-methylbut-2-en-1-yl diphosphate (AMBPP, 12). These inhibitors were designed and synthesized ${ }^{[12,13]}$ and their structures in complex with IspH were released to the Protein Data Bank (entries 3ZGN and 3ZGL, respectively) in 2013. Independently, Span and coworkers reported similar structures (4H4E and 4H4D, respectively). ${ }^{[14]}$ Following detailed analyses of our structures we show that new IspH ligands with prospective improved binding properties can be designed in silico.

\section{Results and Discussion}

The IspH metalloenzyme transforms HMBPP 10 into a mixture of IPP 1 and DMAPP 2 (Scheme 1). The mechanism of this reaction requires reductive removal of the hydroxyl group from the substrate, which is achieved by transfer of two electrons from the [4Fe-4S] cluster and protonation of a formal allyl anion intermediate, which may be stabilized by coordination to iron $\left(\eta^{3}\right.$ allyl $(\pi)$ complex, Scheme $2 \mathrm{~A}) \cdot{ }^{[15,16]}$ The binding of the $\mathrm{OH}$ group in HMBPP 10 to the unique fourth iron site of the [4Fe-4S] cluster was first shown by Mössbauer spectroscopy ${ }^{[7]}$ and later by X-ray crystallography of the E. coli IspH:HMBPP complex. ${ }^{[11]}$ We synthesized two HMBPP analogues where the $\mathrm{OH}$ group was replaced by thiol $(\mathrm{SH})$ or amino $\left(\mathrm{NH}_{2}\right)$, groups expected to

For internal use, please do not delete. Submitted_Manuscript 
coordinate to iron centers. We anticipated that the corresponding derivatives TMBPP 11 and AMBPP 12 (Scheme 2B) would be recognized by the enzyme and bind tightly to the IspH [4Fe-4S] cluster, but may not undergo the elimination. Indeed, TMBPP 11 and AMBPP 12 were potent inhibitors of IspH $\left(\mathrm{IC}_{50}\right.$ values $210 \mathrm{nM}$ and $150 \mathrm{nM}$, respectively ${ }^{[12]}$ and superior to 3-butynyl diphosphate $\left(\mathrm{IC}_{50}=450 \mathrm{nM}\right.$ for the Aquifex aeolicus enzyme). ${ }^{[17]}$ Further kinetic investigations of TMBPP 11 and AMBPP 12 with E. coli IspH and the system NADPH/flavodoxine/flavodoxine reductase, needed for the reduction of the oxidized $[4 \mathrm{Fe}-4 \mathrm{~S}]^{2+}$ cluster, revealed that TMBPP $\mathbf{1 1}$ is a reversible tight-binding inhibitor of IspH $\left(\mathrm{K}_{\mathrm{i}} 20 \mathrm{nM}\right)$ and AMBPP 12 is a reversible slowbinding inhibitor $\left(K_{i} 54 \mathrm{nM}\right) .{ }^{[13]}$ To obtain a clearer insight into the mechanism of inhibition of IspH by 11 and 12, X-ray structures were obtained for the $E$. coli IspH:AMBPP and $E$. coli IspH:TMBPP complexes by molecular replacement using the published E. coli IspH:HMBPP structure as a model (PDB 3KE8). The initial structure (AMBPP, 12) was refined to $1.7 \AA$ and was used to determine the structure of the other form (TMBPP, 11) at $1.95 \AA$ resolution (for crystallization and refinement details see Table 1S, supporting information). Both structures belonged to the same space group (C2) and displayed similar cell parameters. The asymmetric units contained two IspH molecules and have a solvent content of $47.5 \%$. Refined models encompassed residues 1 to 309 and each protein monomer contained one inhibitor molecule and one [4Fe-4S] cluster with an occupancy of $100 \%$. between the unique iron site of the [4Fe-4S] cluster and the nitrogen atom of 12 , or the sulfur atom of 11 was $2.4 \AA$ and $2.3 \AA$, respectively (Figure $1 \mathrm{~B}$ ).

A

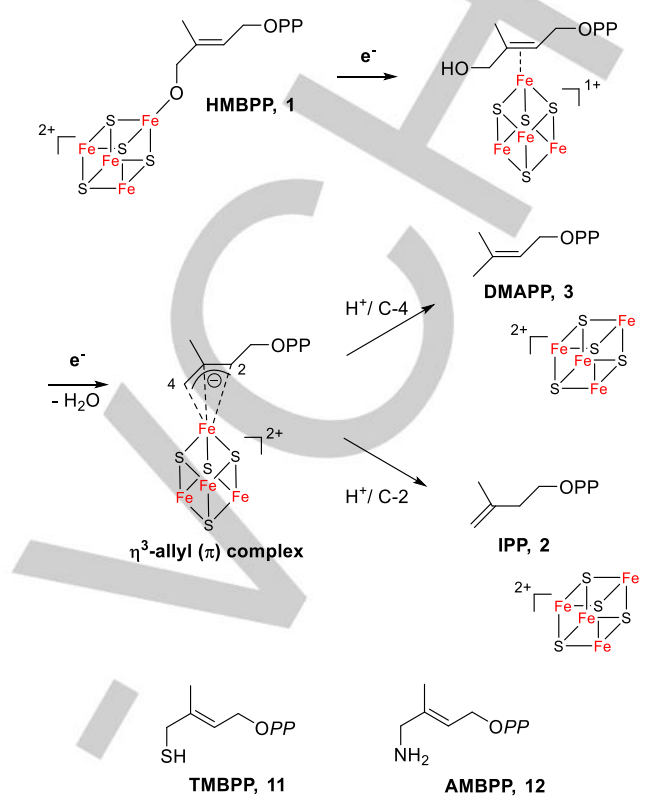

Scheme 2. IspH/LytB catalysis and inhibition: (A) Mechanism of the reaction catalysed by IspH/LytB and (B) structures of the IspH inhibitors TMBPP 11 and AMBPP 12.

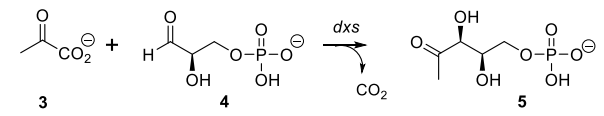$$
\sum_{d x r}^{N A D P H} \text { NADP }^{+}
$$

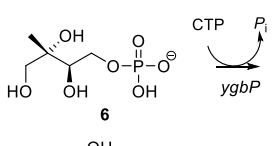

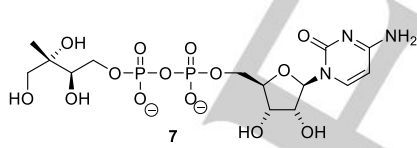

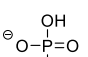

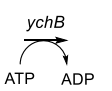

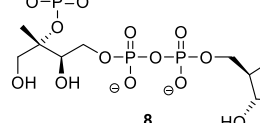

$\mathrm{O}^{-} \mathrm{N}^{-} \mathrm{NH}^{2}$ ygbB

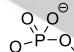

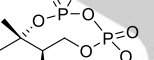

оH CMP

${ }^{2}$

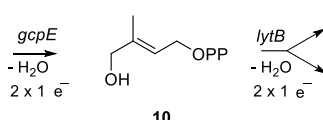

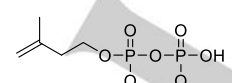

$0_{\theta} 0^{-1}$

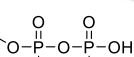

$\mathrm{o}_{\ominus} \mathrm{o}_{\odot}$

2

Scheme 1. Methylerythritol phosphate (MEP) pathway.

Initial inspection indicated that the binding modes of AMBPP 12 and TMBPP 11 are similar to those previously described by the groups of Oldfield and Groll ${ }^{[14]}$ (see however below for 11). As expected from our previously published Mössbauer data for $E$. coli IspH:AMBPP and E. coli IspH:TMBPP complexes, ${ }^{[12]} 12$ binds to the apical iron of the [4Fe-4S] cluster via its amino moiety while 11 binds to this iron via its thiol group. The length of the bond
Interestingly, in the E. coli IspH:TMBPP complex, a careful analysis of the ligand electron densities reveals that the TMBPP bound in each monomer adopted two different conformations (Figure 1B). The TMBPP conformer superimposable on the 'classical' conformer of HMBPP in the $E$. coli IspH:HMBPP complex or on AMBPP 12 in the E. coli IspH:AMBPP complex was the predominant conformer in monomer A. Surprisingly, a second conformer in which the C-4 atom of TMBPP is pulled away from the diphosphate group and from E126, as compared to the 'classical' conformer, was the predominant conformer in monomer B. In the 'classical' conformer, the distances between the C- 4 atom of TMBPP and the nearest oxygen atom attached to the $\beta$-phosphate or $\mathrm{E} 126 \mathrm{O} \varepsilon$ are $3.2 \AA$ and $3.8 \AA$, respectively, while the corresponding distances are $4.7 \AA$ and $5.1 \AA$ for the 'non-classical' conformer (Figure 1B). Importantly, the presence of two TMBPP conformers was overlooked in the previously published structure for the E. coli IspH:TMBPP (PDB 4H4E) complex. ${ }^{[14]}$ However, our analysis of the reported electron density revealed that both TMBPP conformers could be modeled and hence were present in the published structure ${ }^{[14]}$.

The proposed mechanism of the reaction catalyzed by IspH requires initial binding of the $\mathrm{OH}$ group of the substrate to the apical iron of the [4Fe-4S] center. Elimination of the hydroxyl group and electron transfer from the Fe-S cluster yields an allyl anion intermediate coordinated to the cluster. At this point, protonation at C-2 or C-4 yields either IPP 1 or DMAPP 2 (ratio $5: 1$ ) (Scheme $2 \mathrm{~A}$ ). It is still unclear what the proton source is and what parameters influence the regioselectivity of the protonation 

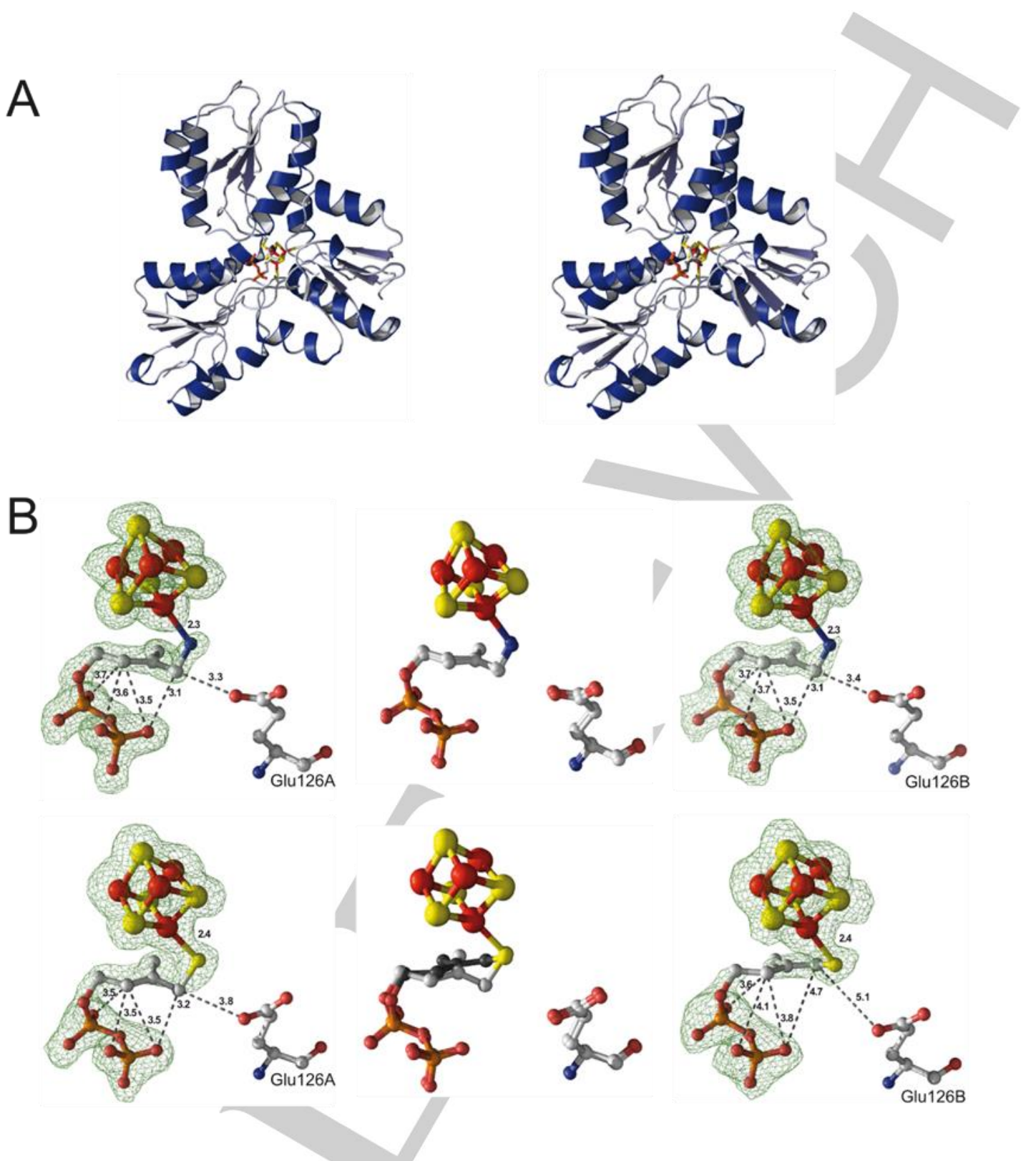

Figure 1. Structure of E. coli LytB/lspH complexed with TMBPP 11 and AMBPP 10. (A) Overall ribbon representation showing the amino (left) and the thio (right) analogues bound to the [4Fe-4S] cluster. (B) Close view of the binding of the two analogues to the [4Fe-4S] cluster (Upper panel IspH:AMBPP; lower panel IspH:TMBPP). Fo-Fc electron density omit maps for the ligands present in each of the two IspH monomer found in the asymmetric unit are contoured at $2.5 \sigma$. Models are depicted as ball and stick representations: right ligand from monomer A; left ligand from monomer B and in between a superimposition of the two. For IspH-TMBPP carbon atoms from the analogue in monomer $B$ are colored in dark gray. Atom distances $(\AA)$ are also indicated.

step. The presence of two conformers of TMBPP in the E. coli IspH:TMBPP complex is puzzling and might be related to the selectivity of the protonation. The IspH enzyme was recently predicted to contain a spacious druggable pocket, formed by binding sites for the substrate and neighboring sites, which might accommodate larger inhibitors than the substrate. ${ }^{[18]}$ Due to the large size of the IspH active site, many conformers of HMBPP 10 might be accommodated and the two conformers observed in the structure of the IspH:TMBPP complex might represent the two most stable when the substrate $\mathbf{1 0}$ is replaced by TMBPP 11. It has been proposed that the hydrogen required for the final protonation could be provided by the $\beta$-phosphate of the allyl anion intermediate. ${ }^{[11,19]}$ As the distance between the C-4 atom of TMBPP and the $\beta$-phosphate differs in both conformers by more than $1 \AA$, it is attractive to propose that protonation at C-4 is disfavored if HMBPP adopts the non-regular conformation, leading instead to protonation at $\mathrm{C}-2$ and hence to the minor product DMAPP 2. The fact that IspH seems to accommodate 
more than one substrate conformer might explain the origin of the two products but this hypothesis needs confirmation.

NRVS data were recently measured for $\mathrm{IspH}$ bound to either TMBPP 11 or AMBPP 12 and compared to the theoretical NRVS data calculated, based on the available crystal structures of IspH in complex with $\mathbf{1 1}$ or $\mathbf{1 2}$. The NRVS data of IspH bound to AMBPP 12 could be calculated using the IspH: AMBPP structure (PDB 3ZGL) presented in this work, but showed significantly less agreement when the IspH:AMBPP (PDB 4H4D) structure was used for the calculation ${ }^{[14]}$. This observation favors the presence in solution of the structure reported here. Two AMBPP conformers were described in the previously reported IspH: AMBPP complex structure (PDB 4H4D) with one AMBPP conformer superimposable on the 'classical' conformer of HMBPP and a second AMBPP conformer with its amino group pulled away from the apical iron of the [4Fe-4S] cluster and rotated by $74^{\circ}$ to generate an interaction with $\mathrm{E} 126 .{ }^{[14]}$ This latter conformer was, however, not included in the PDB file deposited (4H4D) and was surprisingly not observed in the present study. A closer look into the published structure reveals that the Fo-Fc density map presents a significant negative density around the apical iron atom and that the $B$ factors of this iron atom are almost two times higher $(B=17.4 \pm 0.5)$ than the $B$ factors of the other atoms of the [4Fe$4 S$ ] cluster $(B=10.4 \pm 0.8)$. From these observations it seems that the occupancy of the apical iron atoms is lower than $100 \%$. We therefore repeated the refinement of the published structure with various occupancies for the apical iron. Using Refmac5 ${ }^{[20]}$ the best result was obtained for an occupancy of $55 \%$. In that case, the $B$ factors of the apical iron dropped to $12.7 \pm 0.5(10.9 \pm 0.8$ for the other atoms) and neither Fo-Fc positive or negative densities around the apical iron were observed. When using the automated occupancy refinement procedure of Phenix ${ }^{[21]}$ we obtained $66 \%$ and $61 \%$ occupancy for the apical iron of monomer $\mathrm{A}$ and monomer $\mathrm{B}$, respectively. In our opinion, for the structure described in ${ }^{[14]}$ between 35 and $45 \%$ of IspH molecules have an incomplete [4Fe-4S] cluster containing only 3 iron atoms ([3Fe4S]). Therefore, the alternative conformation for AMBPP 12 reported is most probably a result of the absence of the apical iron that led to the formation of an ammonium-carboxylate ion pair with E126 for a fraction of AMBPP in the active site. The lower occupancy of the apical iron in the published structure (PDB 4H4D) may also explain the discrepancies that were observed in the fitting of the NRVS data. ${ }^{[8]}$ A similar second conformation for the substrate 10 in which the $\mathrm{OH}$ interacts with $\mathrm{E} 126$ was also reported ${ }^{[22]}$ in some IspH mutants harboring a [3Fe-4S] center, which also lacked the apical iron. According to these crystallographic studies of IspH in the presence of $\mathrm{AMBPP}^{[14]}$ or IspH mutants in presence of substrate, ${ }^{[22]}$ it was proposed that the mechanism of IspH involves a rotation of almost $180^{\circ}$ of the $\mathrm{CH}_{2} \mathrm{OH}$ group in HMBPP to permit interaction of the $\mathrm{OH}$ group with $\mathrm{E} 126$ in E.coli IspH. The fact that the structure of IspH:AMBPP complex reported here shows no evidence for the formation of an ammonium-carboxylate ion pair with $\mathrm{E} 126$ raises questions about the involvement of E126 in the proposed rotation. According to incorporation experiments of isotopologues of 1deoxy-D-xylulose into pentalenene using Streptomyces avermitilis, it was shown that the formation of IPP $\mathbf{1}$ and DMAPP
2 involves a rotation of the $\mathrm{CH}_{2} \mathrm{OH}$ group of HMBPP. [23] However, it is unclear how this rotation takes place and what the exact role of $\mathrm{E} 126$ is.

\section{LytB inhibitors/better ligands design and evolution:}

The presence of two conformers of TMBPP 11 in the E. coli IspH:TMBPP complex (PDB 3ZGN) can be exploited to design more efficient ligands for $E$. coli IspH. Our objective was to generate putative IspH inhibitors that make additional contacts with the residues of the active site and therefore might display a higher affinity than TMBPP 11 and AMBPP 12. A closer look into the active site of $E$. coli IspH:TMBPP complex revealed that the diphosphate moiety, $\mathrm{C}-1$ and the thiol group of one TMBPP conformer superimpose with the diphosphate moiety, C-1 and the thiol function of the other TMBPP conformer (Figures 1B-2). However, C-2, C-3, C-4 in the aliphatic chains of the conformers are not superimposed in the active site (Figures 1B-2).
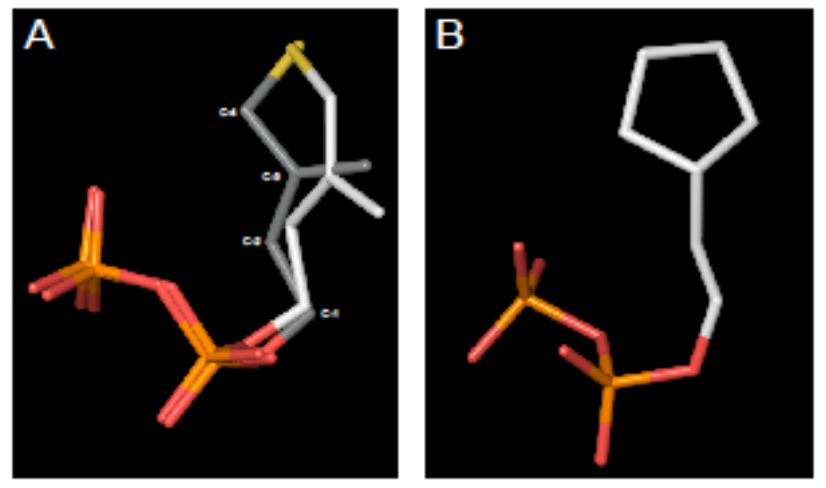

Figure 2. Rational design of new inhibitors/ligands. (A) Superposition of the two TMBPP conformers showing the volume/space available into the active site Ligand from monomer $A$ and monomer $B$ are depicted with grey and white carbon atoms respectively. (B) Cyclopentyl derivative that mimics the volume/space occupied by the two TMBPP conformers. The scaffold of this molecule was use as a starting point for the design of our inhibitor candidates (see Figure 3).

Remarkably, the space occupied by both conformers can be approximately filled by a cyclopentyl group. To take advantage of this observation, we designed a series of molecules to (i) occupy the active site better, (ii) maximize interactions and (iii) provide more rigidity than $\mathbf{1 1}$ or $\mathbf{1 2}$. Our compounds were built around a cyclopentyl scaffold containing amino, hydroxyl, and thiol substituents known to coordinate iron centers and linked to a diphosphate moiety by one or two carbon atoms (Figure 3, column 2). These initial molecules were submitted to a succession of cycles of iterations, in silico docking, and scoring to select the best molecules for the next cycle. The alterations were intended to generate diversity by building a small combinatorial library of slightly different molecules. The differences were introduced with the program Varchem, ${ }^{[24]}$ which generates a large number of molecules by replacing all available hydrogen atoms by methyl, hydroxyl or amino groups. It provides a convenient way to maximize the interactions at the binding site, regardless of the pharmacological properties of the molecules. To validate our

For internal use, please do not delete. Submitted_Manuscript 
docking procedure, some ligands (substrate 10 and inhibitors 11, 12) for which the crystallographic structures in complex with IspH are available, were also submitted to our docking protocol. In each case, the docked ligand superimposed very well with the ligand observed in the crystal structure. The best docking score ${ }^{[25]}$ (D.S $=-7.87$ ) was obtained for AMBPP 12 (Figure 4A).

For the design of new ligands, only molecules that are chemically relevant and displayed docking scores higher than that of AMBPP
12 at the end of the evolution procedure (highest docking score among the molecules used for the validation of the docking procedure) were considered (Figure 3, column 3-5). We generated a series of molecules with excellent docking scores for each of the starting cyclopentyl derivatives. Compound ACA 13 (Figure $4 \mathrm{~B}$ ) is the best ligand (docking score of -10.63) for the series linked to the diphosphate by one carbon atom.

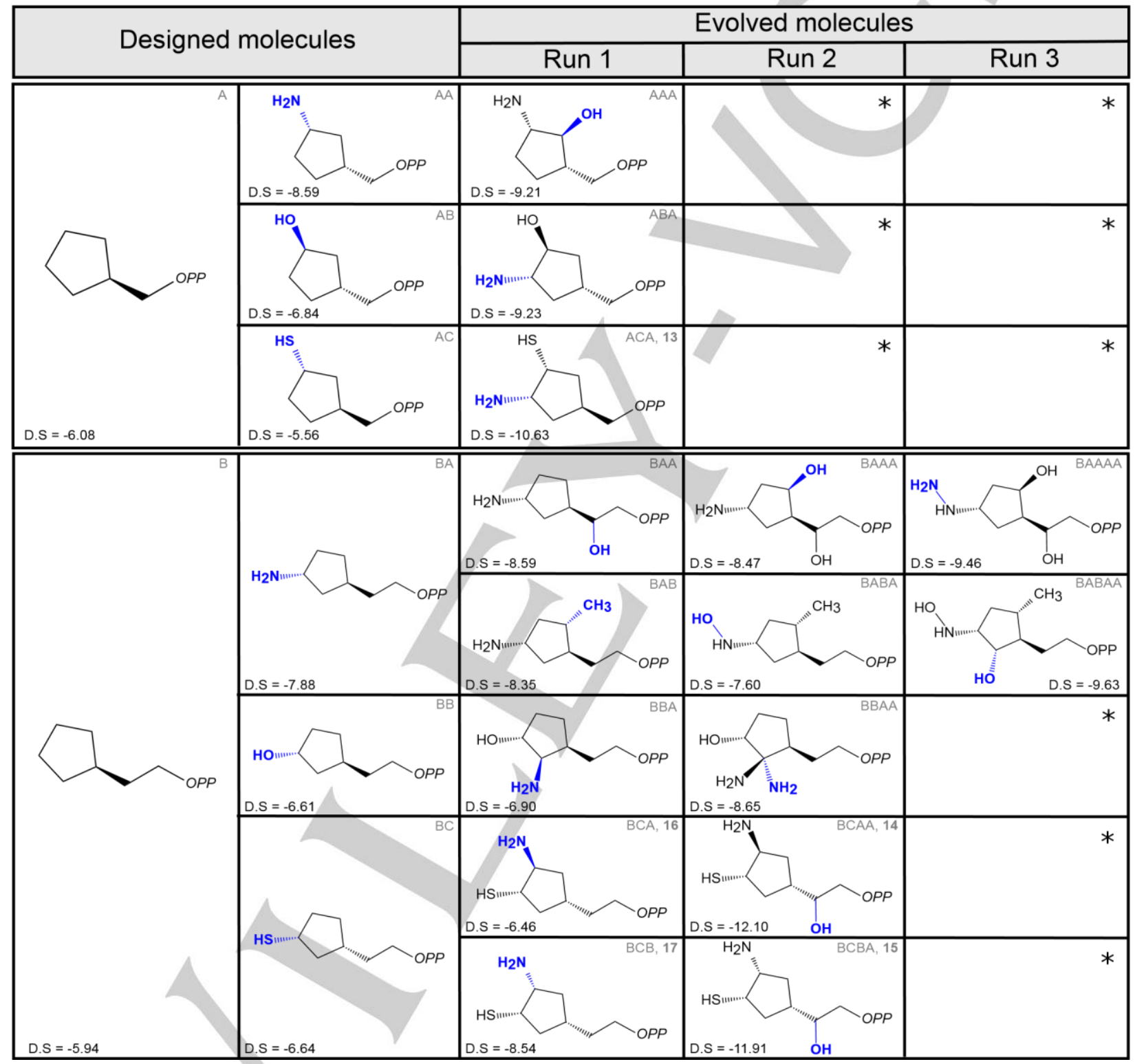

Figure 3. Evolution and docking of the designed IspH inhibitor candidates. Docking experiments were performed using the X-ray structure of $E$. coli IspH:TMBPP complex (PDB: 3ZGL). The inhibitors/ligands were designed based on a cyclopentyl scaffold that was linked to a diphosphate moiety by either one or two carbon atoms and was substituted by either an amino, a hydroxyl or a thiol group (column 1 and 2). Compounds AA-BC were submitted to three successive runs of evolution and in silico docking (column 3-5). The added chemical function after each run of evolution is highlighted with dark blue characters. D.S: docking score. *Docking scores do not improve after evolution. 


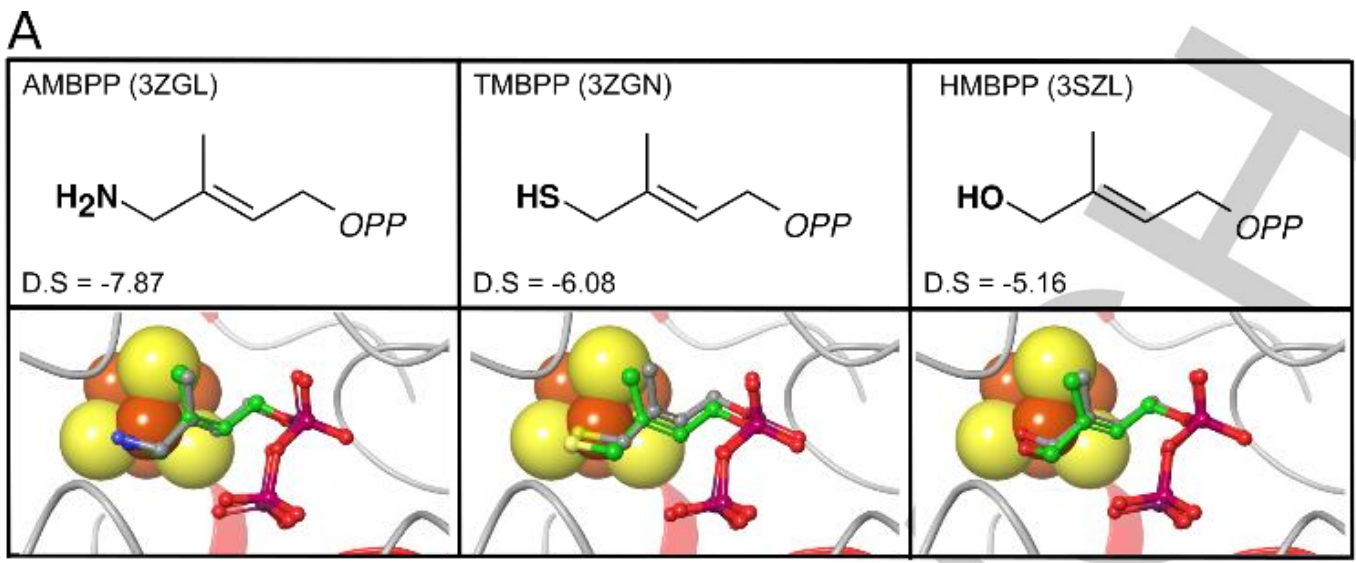

B

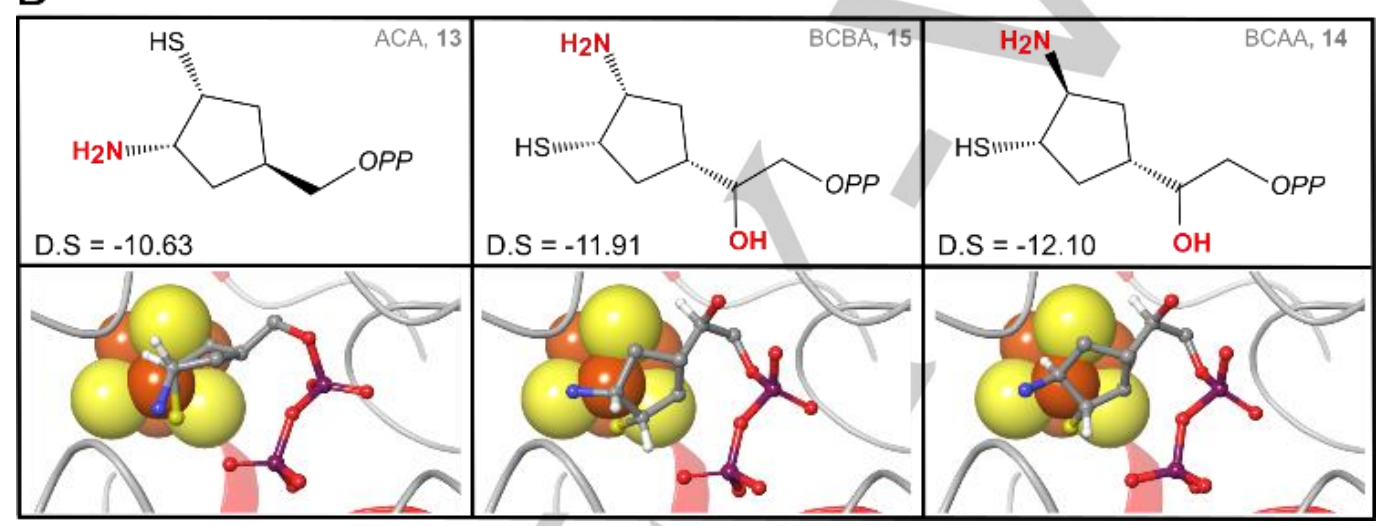

Figure 4. In silico docking results. Docking experiments were performed using the X-ray structure of E. coli IspH:TMBPP complex (PDB: $3 Z$ GL). Upper panel: structural formula and docking score (D.S). Lower panel: 3D arrangement of the compound into IspH active site. (A) Superposition of docked IspH ligands (AMBPP TMBPP, and HMBPP) onto their corresponding crystallographic structure. Docked molecules are depicted with green carbon atoms. (B) The three best ligands/inhibitors arising from the evolution process. The chemical functions added to the initial molecules are highlighted with red characters.

The highest docking scores were obtained with molecules containing a two carbon atom linker. Thus, compound BCAA 14 and its diastereoisomer BCBA 15 (docking scores of -12.10 and 11.91 , respectively) were found to be the best ligands arising from the evolution experiments. Interestingly, ligands ACA 13, BCAA 14 and BCBA 15 contained a cyclopentyl ring substituted with thiol and amino groups on vicinal carbon atoms. Analysis of the position of these three molecules in the IspH active site reveals binding of the thiol moiety to the apical iron in the [4Fe-4S] cluster and formation of a salt bridge between the (protonated) amino group and E126. This latter interaction is not possible for TMBPP 11 or AMBPP 12 and is probably responsible for the improved in silico binding properties of ACA 13, BCAA 14 and BCBA 15. The longer linkers in $\mathbf{1 4}$ and $\mathbf{1 5}$ provide a more flexible side chain that might allow them to adopt a more favorable geometry in the active site. Moreover, when comparing the docking scores of BCA 16, BCB 17 and those of BCAA 14 and BCBA 15 (Figure 3), it appears that addition of a hydroxyl function at $\mathrm{C}-2$ in the linker greatly enhances the affinity of the compounds for the IspH binding pocket. The slight difference observed between the docking scores of the BCAA 14 and BCBA 15 diastereoisomers could be explained by the fact that the conformation of the trans isomer is better adapted for simultaneously coordinating the apical iron of the $[4 \mathrm{Fe}-4 \mathrm{~S}]$ center and forming a salt bridge with the residue E126.

\section{Conclusions}

In summary, our detailed analysis shows that the structures of $E$. coli IspH:AMBPP and E. coli IspH:TMBPP complexes are very similar to the structure of $E$. coli IspH in presence of its substrate and confirms that the amino or thiol moieties of the inhibitors bind to the unique iron of the $[4 \mathrm{Fe}-4 \mathrm{~S}]$ center of IspH. It also reveals for the first time the existence of two different TMBPP conformers. Based on the geometry of these two conformers we were able to design new molecules that should interact simultaneously with the apical iron of the [4Fe-4S] center and with E126. This novel

For internal use, please do not delete. Submitted_Manuscript 
feature gives rise to novel compounds that are attractive targets for synthesis and testing, and presents a new paradigm for the development of more potent IspH inhibitors in the fight against infectious diseases.

\section{Experimental Section}

Cloning, expression and purification: The ispH (lytB) gene of $E$. coli was cloned into $\mathrm{pQE}-30$ (Qiagen) as previously described.[26] The resulting pQE30-LytB plasmid was used to transform E. coli M15 [pREP4] to give $E$. coli M15 strain [pREP4, pQE30-LytB]. E. coli M15 [pREP4, pQE30-LytB] was grown at $30^{\circ} \mathrm{C}$ on LB medium $(6 \times 500 \mathrm{~mL})$ containing ampicillin (100 $\left.\mathrm{mg} \mathrm{mL}^{-1}\right)$ and kanamycin $\left(25 \mathrm{mg} \mathrm{mL}^{-1}\right)$ to $0.6 \mathrm{OD}_{600}$. $\mathrm{FeCl}_{3}(100 \mu \mathrm{M})$ was then added and protein synthesis was induced with IPTG $(100 \mu \mathrm{M})$ for $4 \mathrm{~h}$ at $30^{\circ} \mathrm{C}$. Cells were harvested by centrifugation $(7000 \times \mathrm{g}, 10 \mathrm{~min})$ and kept at $-80^{\circ} \mathrm{C}$ until used.

The following steps were carried out in a glove box (Jacomex BS531 T2) filled with a nitrogen atmosphere containing less than $2 \mathrm{ppm} \mathrm{O}$. E. coll M15 [pREP4, pQE30-LytB] cells (11 g) were suspended in $50 \mathrm{mM}$ Tris$\mathrm{HCl}$ buffer $(20 \mathrm{~mL}, \mathrm{pH} 8)$, and sonicated ( $6 \times 40 \mathrm{~s}$ with $1 \mathrm{~min}$ cooling). After centrifugation $(15000 \times \mathrm{g}, 15 \mathrm{~min})$, the supernatant was collected and loaded onto an $\mathrm{Ni}^{2+}$-NTA column (Qiagen, $1.2 \mathrm{~cm} \times 7 \mathrm{~cm}$ column), equilibrated with $50 \mathrm{mM}$ Tris- $\mathrm{HCl}$ buffer, $\mathrm{pH}$. The resin was first washed with $50 \mathrm{mM}$ Tris- $\mathrm{HCl}$ buffer, $\mathrm{pH} 8$, containing $20 \mathrm{mM}$ imidazole solution followed by same buffer containing $30 \mathrm{mM}$ imidazole $(20 \mathrm{~mL})$. The His 6 IspH protein was eluted with $50 \mathrm{mM}$ Tris- $\mathrm{HCl}$ buffer, $\mathrm{pH} 8(8 \mathrm{~mL})$ containing $100 \mathrm{mM}$ imidazole to give IspH after pooling the most colored and pure fractions (purity $>90 \%$ as judged by SDS-polyacrylamide gel electrophoresis). After concentration on Microcon YM-30 (Millipore), the resulting brown-green protein solution $\left(3 \mathrm{~mL}, 9.5 \mathrm{mg} \mathrm{mL}^{-1}\right)$ was stored in liquid nitrogen.

Crystallization and structure determination: $\mathrm{His} 6-\mathrm{IspH}$ was crystallized under anaerobic conditions by the hanging drop vapor diffusion method at $20^{\circ} \mathrm{C}$ using 24\% PEG 3350, $0.1 \mathrm{M}$ Bis-Tris pH 6.5, $200 \mathrm{mM} \mathrm{LiSO}_{4}$, and a protein concentration of $9.5 \mathrm{mg} \mathrm{mL}^{-1}(260 \mu \mathrm{M})$. Prior to crystallization, the protein was incubated with TMBPP $11(5 \mathrm{mM})$ or AMBPP $12(8 \mathrm{mM})$. Crystals were flash-cooled in liquid propane and transferred to liquid nitrogen when outside the glove box. Native data sets were collected on FIP-BM30A and ID23eh2 beamlines at ESRF (Grenoble, France). The diffraction data were processed using XDS ${ }^{[27]}$. Crystals belong to the $C_{2}$ space group (with unit cell parameters $a=112 \AA, b=80.6 \AA, c=71 \AA, \beta$ $=94.5^{\circ}$ ). The structures were solved by molecular replacement using Phaser ${ }^{[28]}$ and $E$. coli IspH structure (Protein Data Bank (PDB) entry 3KE8) as a search model. Model building was made using $\operatorname{Coot}^{[29]}$ and refinements were carried out with Phenix refine ${ }^{[21]}$ and Refmac5. ${ }^{[20]}$ The stereochemistry of the structure was checked with the programs Procheck ${ }^{[30]}$ and Molprobity. ${ }^{[31]}$ Data and refinement statistics are summarized in table 1S. The coordinates and the structure factors have been deposited in the Protein Data Bank, Research Collaboratory for Structural Bioinformatics, Rutgers University, New Brunswick, NJ (http://www.rcsb.org/) (IspH_TMBPP PDB entry 3ZGN and IspH_AMBPP PDB entry 3ZGL). Figures were generated with PyMOL (The PyMOL Molecular Graphics System, Version 1.8 Schrödinger, LLC).

Docking experiments: Docking experiments were carried out with the Schrödinger suite (Schrödinger, LLC, New York, NY). The X-ray structure of IspH in complex with AMBPP 12 (PDB: 3ZGL) was used for the studies. The protein structure was processed with the Protein Preparation Wizard tool (Epik v2.4, Impact v5.9, and Prime v3.2). The inhibitor AMBPP 12 was used as the reference ligand to generate the grid. The binding region was defined by a square box centered on the inhibitor. Default sizes that are largely exceeding the volume of the binding site were used for both the enclosing and bounding box. LigPrep (v2.6) was used for the ligands energy minimization and to generate their 3D structures. The options "Add metal-binding states" and "include original state" were selected in the Epik interface, all other parameters were set to their default value. Inhibitors were docked into the binding site of IspH using Glide (v5.9) ${ }^{[32]}$ extra precision (XP) mode ${ }^{[25]}$. No constraint was applied to guide the binding. The Glide docking score was used to rank the docking poses.

\section{Acknowledgements}

We are grateful to Prof. Michel Rohmer for helpful discussion. We appreciate the help from the staff of the computing facility provided by the Commissariat à l'Energie Atomique (CEA/DSV/GIPSI), Saclay, France, and the Centre de Calcul Recherche et Technologie (CEA/CCRT), Bruyères-le-Châtel, France. We are grateful for the help from the staff of beamlines FIP-BM30A and ID23-2 at the European Synchrotron Radiation Facility (ESRF, Grenoble, France). We thank Prof. A. Boronat (University of Barcelona, Spain) and his group for providing the $E$. coli strain overexpressing IspH. We are grateful to $\mathrm{M}$. Parisse for technical assistance. This work was supported by the "Agence Nationale de la Recherche" (ANR-05-JCJC-0177-01 and ANR2011-BSV5-028) to M.S. and J.-L. F., by the Thai government to K.J., by COST Action CM1201 to M.S and by NIH grant GM21328 to C.D.P.

Keywords: MEP pathway inhibitors $\cdot$ LytB/lspH $\cdot[4 \mathrm{Fe}-4 \mathrm{~S}]$

cluster $\cdot$ X-ray crystallography $\bullet$ In silico docking

[1] K. Bloch, Steroids 1992, 57, 378-383

[2] M. Rohmer, Nat. Prod. Rep. 1999, 16, 565-574.

[3] W. Eisenreich, F. Rohdich, A. Bacher, Trends Plant Sci. 2001, 6 , 78-84.

[4] M. Rohmer, C. Grosdemange-Billiard, M. Seemann, D. Tritsch, Curr. Opin. Investig. Drugs Lond. Engl. 2000 2004, 5, 154-162.

[5] T. Gräwert, M. Groll, F. Rohdich, A. Bacher, W. Eisenreich, Cell. Mol. Life Sci. 2011, 68, 3797-3814.

[6] T. Masini, A. K. H. Hirsch, J. Med. Chem. 2014, 57, 9740-9763.

[7] M. Seemann, K. Janthawornpong, J. Schweizer, L. H. Böttger, A. Janoschka, A. Ahrens-Botzong, E. N. Tambou, O. Rotthaus, A. X Trautwein, M. Rohmer, V. Schünemann, J. Am. Chem. Soc. 2009 131, 13184-13185.

[8] I. Faus, A. Reinhard, S. Rackwitz, J. A. Wolny, K. Schlage, H.-C Wille, A. Chumakov, S. Krasutsky, P. Chaignon, C. D. Poulter, M. Seemann, V. Schünemann, Angew. Chem. Int. Ed Engl. 2015, 54 12584-12587.

[9] I. Rekittke, J. Wiesner, R. Röhrich, U. Demmer, E. Warkentin, W Xu, K. Troschke, M. Hintz, J. H. No, E. C. Duin, E. Oldfield, H. Jomaa,, U. Ermler, J. Am. Chem. Soc. 2008, 130, 17206-17207.

[10] T. Gräwert, F. Rohdich, I. Span, A. Bacher, W. Eisenreich, J. Eppinger, M. Groll, Angew. Chem. Int. Ed. 2009, 48, 5756-5759.

[11] T. Gräwert, I. Span, W. Eisenreich, F. Rohdich, J. Eppinger, A. Bacher, M. Groll, Proc. Natl. Acad. Sci. U. S. A. 2010, 107, 10771081.

[12] A. Ahrens-Botzong, K. Janthawornpong, J. A. Wolny, E. N. Tambou, M. Rohmer, S. Krasutsky, C. D. Poulter, V. Schünemann M. Seemann, Angew. Chem. Int. Ed Engl. 2011, 50, 11976-11979.

[13] K. Janthawornpong, S. Krasutsky, P. Chaignon, M. Rohmer, C. D. Poulter, M. Seemann, J. Am. Chem. Soc. 2013, 135, 1816-1822.

[14] I. Span, K. Wang, W. Wang, J. Jauch, W. Eisenreich, A. Bacher E. Oldfield, M. Groll, Angew. Chem. Int. Ed Engl. 2013, 52, 2118 2121.

For internal use, please do not delete. Submitted_Manuscript 
[15] R. Laupitz, T. Gräwert, C. Rieder, F. Zepeck, A. Bacher, D. Arigoni, F. Rohdich, W. Eisenreich, Chem. Biodivers. 2004, 1, 1367-1376.

[16] W. Wang, K. Wang, I. Span, J. Jauch, A. Bacher, M. Groll, E. Oldfield, J. Am. Chem. Soc. 2012, 134, 11225-11234.

[17] K. Wang, W. Wang, J.-H. No, Y. Zhang, Y. Zhang, E. Oldfield, J. Am. Chem. Soc. 2010, 132, 6719-6727.

[18] T. Masini, B. S. Kroezen, A. K. H. Hirsch, Drug Discov. Today 2013 18, 1256-1262.

[19] A. Frank, M. Groll, Chem. Rev. 2017, 117, 5675-5703.

[20] A. A. Vagin, R. A. Steiner, A. A. Lebedev, L. Potterton, S. McNicholas, F. Long, G. N. Murshudov, Acta Crystallogr. D Biol. Crystallogr. 2004, 60, 2184-2195.

[21] P. V. Afonine, R. W. Grosse-Kunstleve, N. Echols, J. J. Headd, N. W. Moriarty, M. Mustyakimov, T. C. Terwilliger, A. Urzhumtsev, P. H. Zwart, P. D. Adams, Acta Crystallogr. D Biol. Crystallogr. 2012, 68, 352-367.

[22] I. Span, K. Wang, W. Wang, Y. Zhang, A. Bacher, W. Eisenreich K. Li, C. Schulz, E. Oldfield, M. Groll, Nat. Commun. 2012, 3, 1042.

[23] C. A. Citron, N. L. Brock, P. Rabe, J. S. Dickschat, Angew. Chem. Int. Ed. 2012, 51, 4053-4057.

[24] J.-L. Ferrer, J. Dupuy, F. Borel, L. Jacquamet, J. P. Noel, V. Dulic, Cell Cycle 2006, 5, 2760-2768.
[25] T. A. Halgren, R. B. Murphy, R. A. Friesner, H. S. Beard, L. L. Frye W. T. Pollard, J. L. Banks, J. Med. Chem. 2004, 47, 1750-1759.

[26] M. Wolff, M. Seemann, B. Tse Sum Bui, Y. Frapart, D. Tritsch, A. G. Estrabot, M. Rodríguez-Concepción, A. Boronat, A. Marquet, M. Rohmer, FEBS Lett. 2003, 541, 115-120.

[27] W. Kabsch, Acta Crystallogr. D Biol. Crystallogr. 2010, 66, 125132.

[28] A. J. McCoy, R. W. Grosse-Kunstleve, P. D. Adams, M. D. Winn L. C. Storoni, R. J. Read, J. Appl. Crystallogr. 2007, 40, 658-674.

[29] P. Emsley, B. Lohkamp, W. G. Scott, K. Cowtan, Acta Crystallogr. D Biol. Crystallogr. 2010, 66, 486-501.

[30] R. A. Laskowski, D. S. Moss, J. M. Thornton, J. Mol. Biol. 1993 231, 1049-1067.

[31] V. B. Chen, W. B. Arendall, J. J. Headd, D. A. Keedy, R. M. Immormino, G. J. Kapral, L. W. Murray, J. S. Richardson, D. C. Richardson, Acta Crystallogr. D Biol. Crystallogr. 2010, 66, 12-21.

[32] R. A. Friesner, R. B. Murphy, M. P. Repasky, L. L. Frye, J. R Greenwood, T. A. Halgren, P. C. Sanschagrin, D. T. Mainz, J. Med. Chem. 2006, 49, 6177-6196. 
Entry for the Table of Contents (Please choose one layout)

Layout 1:

\section{FULL PAPER}

Thanks to the crystal structures of Escherichia coli IspH in complex with (E)-4-mercapto-3-methylbut-2-en-1-yl diphosphate and $(E)$-4-amino-3methylbut-2-en-1-yl diphosphate we were able to design IspH ligands with improved binding capacities. This novel binding feature gives rise to compounds that present a new paradigm for the development of more potent IspH inhibitors.

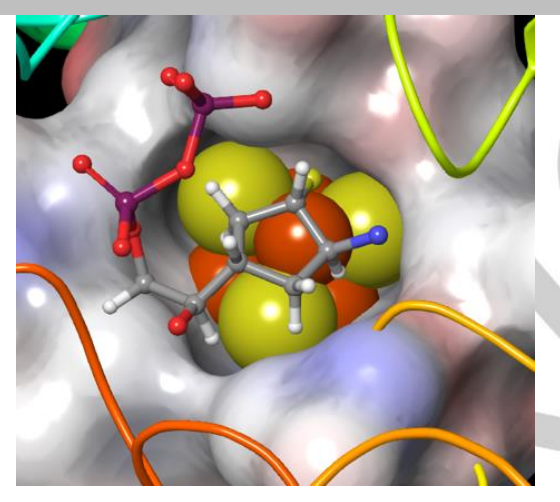

Franck Borel*, Elodie Barbier, Sergiy Krasutsky, Karnjapan Janthawornpong, Philippe Chaignon, C. Dale Poulter, Jean-Luc Ferrer and Myriam Seemann*

Page No. - Page No.

Further insight into crystal structures of $E$. coli IspH/LytB in complex with two potent inhibitors of the MEP pathway: a starting point for rational design of new antimicrobials

Title

For internal use, please do not delete. Submitted_Manuscript 